Originally published as:

Korte, M., Constable, C. G., Parker, R. L. (2002): Revised magnetic power spectrum of the oceanic crust. - JOURNAL OF GEOPHYSICAL RESEARCH, 107, B9

DOI:10.1029/2001JB001389

http://www.agu.org/pubs/crossref/2002/2001JB001389.shtml 


\title{
Revised magnetic power spectrum of the oceanic crust
}

\author{
M. Korte, C. G. Constable and R. L. Parker \\ Institute of Geophysics and Planetary Physics, Scripps Institution of Oceanography, University of California, \\ San Diego, La Jolla, USA \\ Received 24 September 2001; revised 14 March 2002; accepted 19 March 2002; published XX Month 2002.
}

[1] The magnetic field originating within the Earth can be divided into core and crustal components, which can be characterized by the geomagnetic power spectrum. While the core spectrum is determined quite well by satellite studies, models of the shorter wavelength crustal spectrum disagree considerably. We reexamine aeromagnetic data used by O'Brien et al. [1999] to obtain a new, improved estimate of the crustal geomagnetic power spectrum. O'Brien et al.'s model somewhat failed to give a satisfactory connection between the longer-wavelength satellite studies and a reliable crustal model. We show that this was caused by an inadequate processing step that aimed to remove external variations from the data. We moreover attempt to bound the long-wavelength part of the spectrum using constraints of monotonicity in the correlation of the magnetization. However, this proves to be a weak constraint. Reversing the process, though, we are able to evaluate the correlation function using the reliable part of our geomagnetic spectrum. Thus we can obtain a sensible estimate for the long-wavelength part of the spectrum that is not well constrained by the data. Our new model shows better agreement with earlier satellite studies and can be considered reliable in the spherical harmonic degree range $l=$ 30 to 1200. INDEX TERMS: 1532 Geomagnetism and Paleomagnetism: Reference fields (regional, global); 1545 Geomagnetism and Paleomagnetism: Spatial variations (all harmonics and anomalies); 1599 Geomagnetism and Paleomagnetism: General or miscellaneous; KEYWORDS: geomagnetic power spectrum, crustal magnetization, aeromagnetic data, geomagnetic stochastic process

Citation: Korte, M., C. G. Constable, and R. L. Parker, Revised magnetic power spectrum of the oceanic crust, J. Geophys. Res., 107(0), XXXX, doi:10.1029/2001JB001389, 2002.

\section{Introduction}

[2] Over the last decades several models of the geomagnetic spectrum of the Earth have been developed, both from satellite observations and theoretical considerations. There is a satisfying agreement about the long-wavelength part of the spectrum, originating mainly in the Earth's core. For spherical harmonic degrees larger than about $l=15$, however, the different models disagree considerably. Knowledge of this part of the spectrum is useful to estimate the depth of the crustal magnetization and its length scale of correlation [Jackson, 1990, 1994]. Estimates of the main field from satellite measurements can be improved by a quantitative estimate of the crustal spectrum [RygaardHjalsted et al., 1997]. A recent study based on large scale aeromagnetic data by O'Brien et al. [1999] offers an extension in spherical harmonic degree of the observational based models from $l \approx 60$ of the satellite studies to $l=1200$. The agreement of this model for degrees $l<100$ with the satellite models, however, is somewhat unsatisfactory. We believe the reason for this disagreement lies mainly in the inappropriate treatment of the influence of external magnetic variations in the data. For the analysis the pure crustal

Copyright 2002 by the American Geophysical Union. 0148-0227/02/2001JB001389\$09.00 magnetic field is needed, and therefore the main field part and the influence of external variations have to be removed from the raw data. We improve this processing step in order to obtain a better model at the long wavelengths.

[3] A variety of geomagnetic spectral estimates are used for comparison with our new model, namely, those by Cain et al. [1989], Ravat et al. [1995], Lowe et al. [2001], Jackson [1994], and Langel et al. [1989]. The Cain et al. [1989] and Ravat et al. [1995] models are based on spherical harmonic analyses of Magsat data, Cain et al.'s model includes the core field, while Ravat et al.'s just considers the crustal field. Lowe et al. [2001] also use Magsat data but follow the approach of O'Brien et al. [1999], namely, to isolate the crustal anomalies from the data, estimate power spectral densities and invert those for the geomagnetic spectrum. The same method is adopted for the current work. The last two models are theoretical ones. Jackson [1994] employed considerations of correlation scales in the crustal magnetization and adjusted his model parameters to fit Cain's empirical estimate and to give a suitable RMS value of the lithospheric field as observed in observatory biases [Bloxham et al., 1989]. The model by Langel et al. [1989] obeys a simple power law, derived also using Cain's model results and correlation considerations.

[4] Another different approach to obtain a crustal spectrum is followed by Meyer et al. [1983]. They set up a 
model of the crust consisting of 32,400 blocks with the magnetic effect of each block approximated by a dipole. Considering only induced magnetization, the parameters of the blocks were adjusted so that the magnetic field from this model fit the Magsat magnetic anomaly field. Then Meyer et al. [1983] calculated a geomagnetic spectrum for this crust model by spherical harmonic analysis up to degree 35 . After a steep rise in the first 4 spherical harmonic degrees this white spectrum agrees to first order with that of Langel et al. [1989]. Because of the minimal overlap of this spectrum with the reliable part of our spectrum which starts at spherical harmonic degree 30 we did not include it in our direct comparison.

\section{Power Spectral Densities and Geomagnetic Spectra}

[5] We use the method given by O'Brien et al. [1999] (based on an approach by McLeod and Coleman [1980]) to obtain power spectral densities (PSD) and geomagnetic spectra from statistical properties of magnetic fields measured on segments of great circles. More details of the theory together with the necessary assumptions to derive the linear relationships between the PSDs and the geomagnetic spectra are given by O'Brien et al. [1999]. The Earth's crustal magnetic field is considered to be one particular realization of a stationary, stochastic process with complete rotational symmetry. The latter allows every great circle to be treated as equivalent and the use of local track parallel and track perpendicular coordinates. The statistical properties depend only on the distance between two observation points, not on the direction.

[6] The geomagnetic power spectrum, $R_{l}$, is defined as the mean square value of the magnetic field's magnitude per spherical harmonic degree $l$. Using the Schmidt seminormalized Gauss coefficients $\left(g_{l}^{m}, h_{l}^{m}\right), R_{l}$ can be written as

$$
R_{l}=(l+1) \sum_{m=0}^{l}\left(g_{l}^{m}\right)^{2}+\left(h_{l}^{m}\right)^{2} .
$$

The relations between $R_{l}$ and the power spectral densities for the parallel to track $\left(P_{x x}(k)\right)$, perpendicular to track $\left(P_{y y}(k)\right)$, and radial $\left(P_{z z}(k)\right)$ component, as well as for the cross spectral density $P_{x z}(k)$ are given by

$$
\begin{gathered}
P_{x x}(k)=4 \pi r_{0} \sum_{l=|m|}^{\infty} \frac{m^{2} R_{l}}{(2 l+1)(l+1)}\left(\frac{a}{r_{0}}\right)^{2 l+4} \tilde{P}_{l}^{m}(0)^{2} \\
P_{y y}(k)=4 \pi r_{0} \sum_{l=|m|}^{\infty} \frac{R_{l}}{(2 l+1)(l+1)}\left(\frac{a}{r_{0}}\right)^{2 l+4} \tilde{D}_{l}^{m}(0)^{2} \\
P_{z z}(k)=4 \pi r_{0} \sum_{l=|m|}^{\infty} \frac{(l+1) R_{l}}{(2 l+1)}\left(\frac{a}{r_{0}}\right)^{2 l+4} \tilde{P}_{l}^{m}(0)^{2} \\
P_{x z}(k)=4 \pi r_{0} \sum_{l=|m|}^{\infty} \frac{i m R_{l}}{(2 l+1)}\left(\frac{a}{r_{0}}\right)^{2 l+4} \tilde{P}_{l}^{m}(0)^{2} .
\end{gathered}
$$

Here, $k=m / 2 \pi r_{0}$ is the wave number, $r_{0}$ is the mean radius of the Earth $(6371.2 \mathrm{~km})$, and $r$ is the geocentric radius of the measurements. The $l$ and $m_{\tilde{P}}$ are spherical harmonic degree and order, respectively; $\tilde{P}_{l}^{m}(\mu)$ are the associated Legendre functions; and $\tilde{D}_{l}^{m}(\mu)$ are their derivatives with respect to $\mu=\cos \theta, \theta$ being the colatitude. The $P_{x z}$ cross spectrum is purely imaginary, its phase for internal signals should be $\pi / 2$. This property and the power sum rule $P_{x x}+$ $P_{y y}=P_{z z}$ [Parker and O'Brien, 1997] are used to confirm the agreement between the data and the assumptions made to derive the equations. The theory predicts the coherence and thus the cross spectral densities between other pairs of components to be zero.

\section{Data Processing}

[7] We reexamined the Project Magnet data used by O'Brien et al. [1999]. The data of this global aeromagnetic field mapping mission flown between 1953 and 1994 are available on CD-ROM from the National Geophysical Data Center. Details of the mission and the data are given in the accompanying manual by Hittelmann et al. [1996]. The 17 profiles were selected for tracks of maximum length and good approximate arcs of great circles. The deviation of the chosen paths from exact great circles is remarkably slight, usually smaller than $10 \mathrm{~km}$. We directly use the cleaned up, edited paths of O'Brien et al. [1999]. Figure 1 gives an overview over the edited tracks. The lengths range from 3682 to $6824 \mathrm{~km}$, the average being $5583 \mathrm{~km}$. They were measured at heights between 6847 and $8278 \mathrm{~m}$. In the following calculations we use the mean altitude of $7520 \mathrm{~m}$. For more details on the raw data see O'Brien et al. [1999].

[8] The preparation of the data to calculate the estimates of the great circle Fourier spectra follows the seven steps described by O'Brien et al. [1999], except for the treatment of the main and external field correction (steps 4 and 6). Those parts of the magnetic field have to be eliminated from the measurements to get the crustal anomalies we are interested in. We initially intended to improve the correction for external variations by using the comprehensive nearEarth magnetic field model (CMP3) by Sabaka et al. [2000]. This model has been derived using satellite and ground observations to represent not only the core and magnetospheric field but also all other contributions from ionosphere, induction in the lithosphere, and coupling currents between ionosphere and magnetosphere and between the hemispheres. The contributions of the different sources may be calculated separately by the model; the spherical harmonic expansion up to degree 65 includes crustal fields as measured at satellite altitude. The original CMP3 model only covers the time span from 1960 to 1985 , using the POGO and Magsat satellite missions, but does not include the time over which the aeromagnetic data are distributed, 1984 to 1994 . There is an updated version of CMP3, referred to as CMP31, which includes data from the Oersted satellite and thus goes to 2000 . The primary intent of this update, however, was to check whether the Oersted quiet time external data are consistent with those of POGO and Magsat. Therefore at the time of our study there was little data control and no smoothing for the secular variation between the Magsat and Oersted satellite epochs (T. J. Sabaka, personal communication, 2001). As this leads to strong artificial oscillations of the main field part of the model, we cannot use it for the main field correction to our 


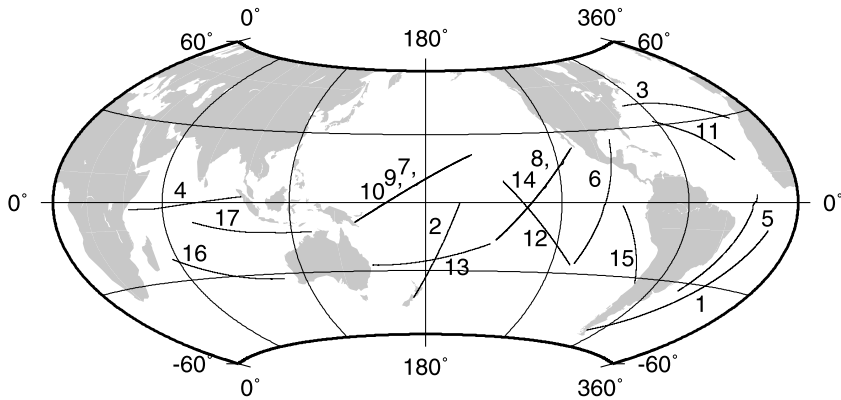

Figure 1. Edited Project Magnet flight paths used in our study.

data. However, the external parts of the model give a good representation of the external fields for the whole time span, as we verified by comparing the model predictions to observatory data. We decided to use CMP31 for the correction for the external parts and a separate model for the main field. We chose the GSFC model [Sabaka et al., 1997] instead of the IGRF for that task for two reasons: First, the field change with time is represented by cubic B splines in the GSFC model, giving a smoother representation of secular variation than a linear interpolation of the IGRF. Second, the spherical harmonic expansion goes up to degree 13 in the GSFC model, representing the main field more completely than the IGRF, which only goes up to degree 10 . Although we might take out very long wavelength crustal contributions as well, this should not be a problem as we could not resolve anomalies on that scale with our data anyway. Spherical harmonic degree 13 corresponds to a wavelength of $3080 \mathrm{~km}$, and the usable average length of the aeromagnetic flight paths is less than $6000 \mathrm{~km}$. The GSFC model also allows the inclusion of the magnetospheric field of degree 1 , but we, of course, only used the internal model.

[9] The core field along each flight path is calculated by the GSFC model. Also, the sum of all external and induced field contributions is calculated by the CMP31 model. Both model fields are subtracted from the data. Then the horizontal field for each path is projected onto the local trackparallel and track-perpendicular coordinates of the best fitting great circle (step 5 of O'Brien et al. [1999]). O'Brien et al. [1999] removed a best fitting cubic polynomial as representing the external field contributions at this stage. Although we also still see very long wavelength effects in the data, we propose that this is either real crustal signal or unremoved remainders of the core field. This assumption is supported by the comparison of the resulting anomalies for the two flight tracks which have been covered two and three times, respectively (tracks 8 and 14 and 7, 9, and 10 in Figure 1); see Figure 2. The repetition occurred in different years and at different times of the day, yet the anomaly data agree very well both in short-wavelength anomalies and long-wavelength features. However, we remove a linear trend from the data to reduce the bias in the PSD estimations. Finally, the data are resampled by linear interpolation to a regular spacing of $350 \mathrm{~m}$, close to the original average sampling interval, which was uniform in time. Figure 3 shows an average example of differences between our final crustal anomaly data and that of O'Brien et al. [1999]. While they completely agree in the short-wavelength features, there is more long-wavelength structure left in our data.

[10] Now the power and cross spectral densities can be estimated. Again we use the procedure described in detail by O'Brien et al. [1999] and Parker and O'Brien [1997]. It is a multitaper estimation based on sine weight functions [Riedel and Sidorenko, 1995]. A different number of tapers is applied in each frequency band to obtain an optimal balance between bias and variance at each frequency. We are interested in the longer wavelengths, so we need a good frequency resolution and should not smooth the spectral densities excessively. However, rather smooth spectra are obtained when averaging the spectra of the individual tracks. Generally, our PSDs look quite similar to O'Brien et al.'s Figure 5. We screen the data for consistency with the power sum rule and the phase criterion. We consider the power sum rule as satisfied when the error bars determined in estimating the spectra for each frequency overlap for $P_{z z}$ and $P_{x x}+P_{y y}$, using Gauss' law of error propagation for the error of the sum. The power sum rule is satisfied for almost all the data in the wave number range from 0.001 to 0.03 or $0.04 \mathrm{~km}^{-1}$. Only some tracks are satisfactory for larger or smaller wave numbers. Almost the same is true for the phase and coherence of the cross spectral densities, with those criteria often being well satisfied for only slightly higher wave numbers than $0.001 \mathrm{~km}^{-1}$. We decided to consider the range up to
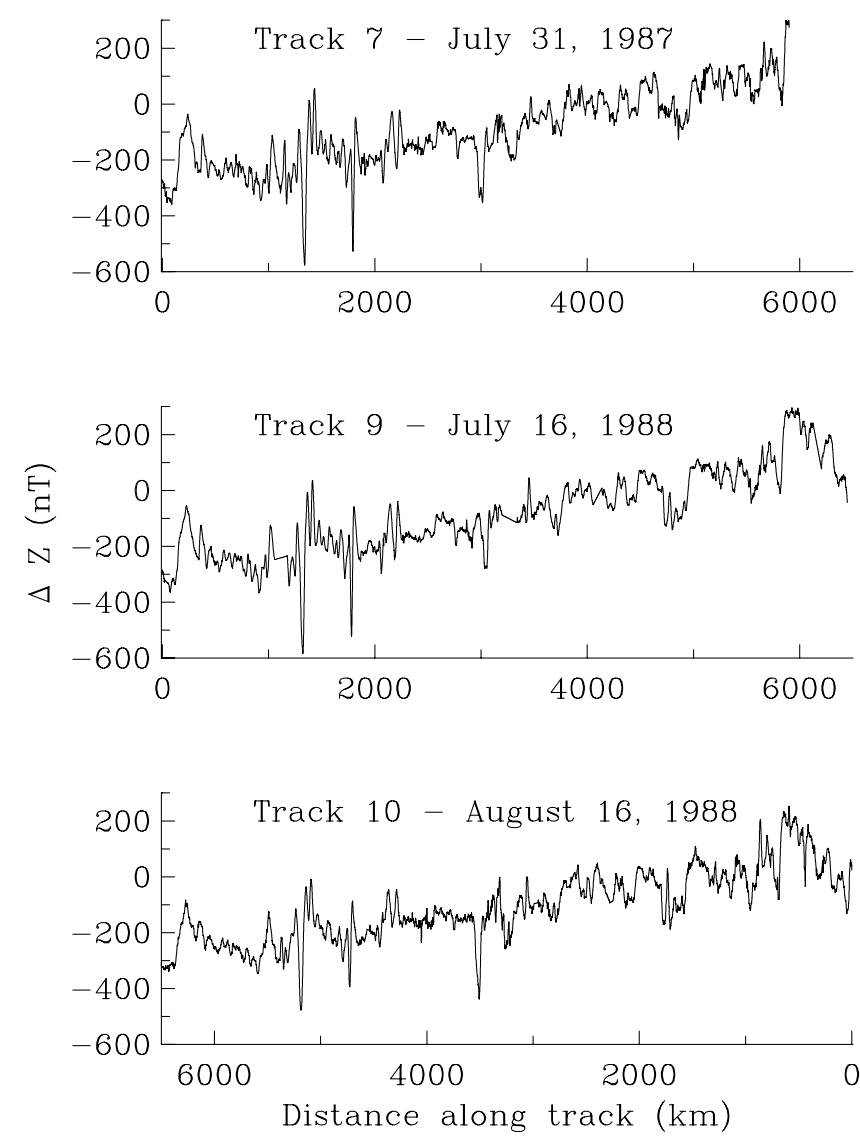

Figure 2. Crustal anomalies of the $\mathrm{Z}$ component for a repeated track. The one named track 10 has been flown in the opposite direction of the other two. Starting and end points are very close but not exactly the same. 


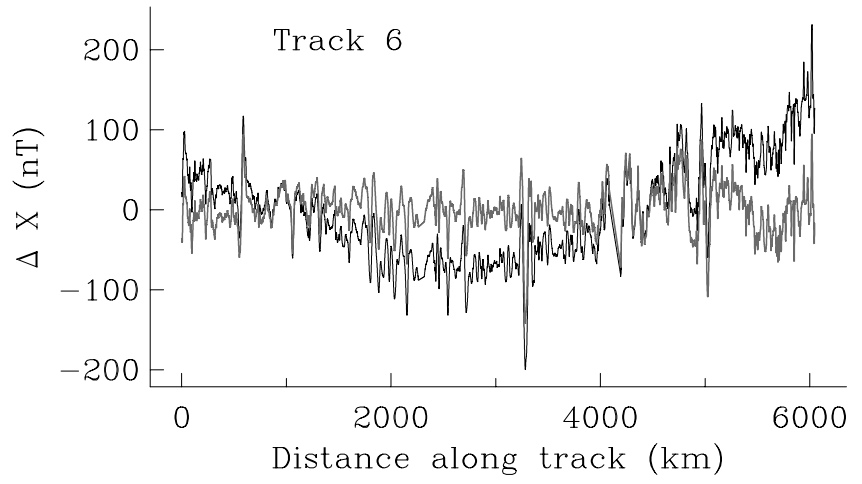

Figure 3. Comparison between crustal anomaly data of the $\mathrm{X}$ component of one flight track as obtained by our processing (black) and that of O'Brien et al. [1999] (gray).

$0.03 \mathrm{~km}^{-1}$, corresponding to wavelengths down to about 30 $\mathrm{km}$. All frequencies within that range where the power sum rule is not satisfied are rejected, at the same time rejecting the corresponding cross spectral densities.

[11] The different lengths of the data series cause different sampling intervals of wave number in the individual spectra. Therefore we have to average the data in wave number bins. We adopt O'Brien et al.'s [1999] bin size of $0.001 \mathrm{~km}^{-1}$, which admits enough samples for error reduction without incurring bias from curvature. The first bin is centered at $0.0005 \mathrm{~km}^{-1}$. Figure 4 shows the bin averaged cross spectral densities of all tracks. There is a clear improvement in these cross spectral densities: O'Brien et al. had to discard the data from the first two bins and still the data in the long-wavelength bins of the cross spectral densities showed significantly less power than those in the PSDs. Now, the PSDs and the cross spectral densities look very much alike, proving that they are more internally consistent, with our PSDs also having slightly more power in the small wave numbers. We treat the individual flight track spectral densities as independent samples of the stochastic process and average them together. Uncertainties for those values are estimated by the scatter in each frequency bin. Figure 5 shows the averages including the estimated error bars.

\section{Inversion to the Geomagnetic Spectrum}

[12] The inversion of the PSDs to the geomagnetic spectrum also follows the approach of O'Brien et al. [1999]. The optimally fitting solution is found by nonnegative least squares (NNLS) [Lawson and Hanson, 1974]:

$$
\min _{\mathbf{x} \geq 0}\|A \mathbf{x}-\mathbf{d}\|_{2}^{2} .
$$

The matrix $A$ consists of the factors relating the vector $\mathbf{x}$ of $R_{l}$ to the data vector $\mathbf{d}$ consisting of the PSDs according to equations (2) to (5). By weighting the equations with the inverse of the standard errors, the squared norm is just the $\chi^{2}$ statistic. This should be of the order of the number of data to obtain a reasonable model that fits the data within the uncertainties. In contrast to O'Brien et al. [1999], we can fit our data very well by this method, the minimum $\chi^{2}$ is 7.4 while the number of data is 120 . The predictions from this best fit model are shown by the thin line in Figure 5 . Obviously, this model seriously overfits the data. We are looking for a solution as smooth as the data allow within the uncertainties. Therefore we do a regularized inversion as described in detail by Parker [1994]. With a Lagrange multiplier we minimize the roughness of the model defined by the 2-norm of its second derivative over the set of models fitting the data with $\chi^{2}=N, N=120$ being the number of data constraints. The resulting model is displayed in Figure 6, and the corresponding fit to the data is also included in Figure 5 as the thick line. The model is constrained by the spectral estimates for the spherical harmonic degrees $l=20$ to 1200 , for larger degrees it is shaped by the smoothness demand and the maximum number of model parameters. Our $R_{l}$ model has remarkably more power in the long wavelengths ( $l$ up to about 300) than O'Brien et al.'s, thus agreeing better with earlier satellite and theoretical models in the long-wavelength part (see Figure 8). However, our model in Figure 6 displays substantially too much power in the very long wavelengths, which are not directly constrained by the spectral estimates from the data. If the main field model we have taken out is a very good representation of the Earth's magnetic field, we should have removed all power up to degree 13 from our data, even if these low degrees contained power from very long wavelength crustal anomalies. Moreover, by the limited length of the aeromagnetic flight paths, the spectral estimates cannot contain power for the very long wavelengths. The model spectrum in that part is dictated only by the smoothness constraint, which makes it flat in the range of $l=20$ down to $l=0$. In section 5 we try to improve this unlikely feature of the model.

\section{Constraints on the Long-Wavelength Spectrum}

[13] Jackson [1996] tried to bound the long-wavelength crustal magnetic field of a stochastic model by using a

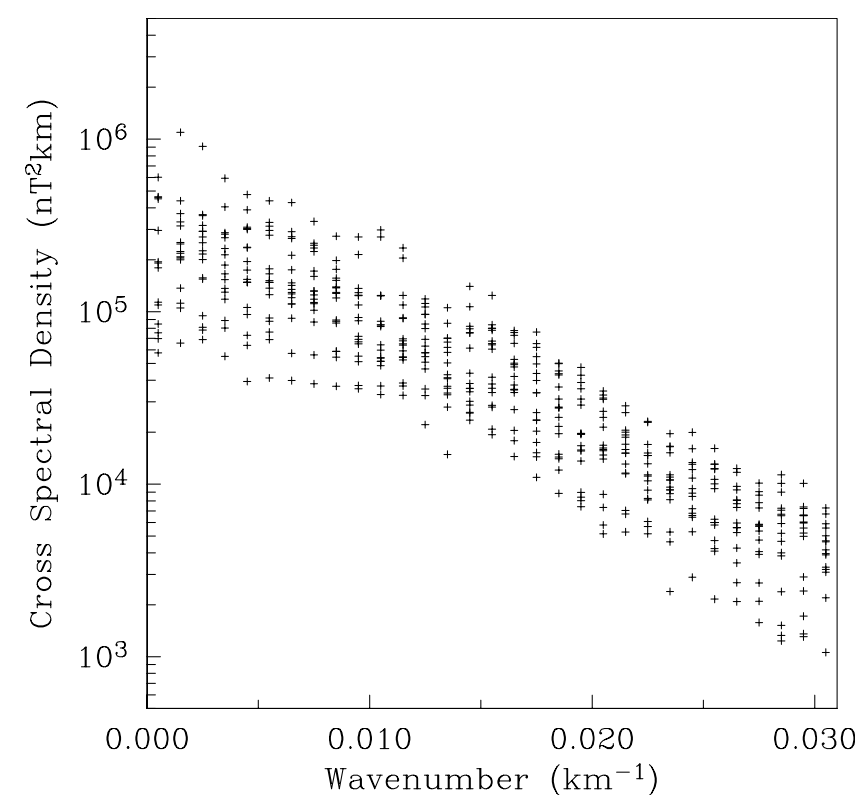

Figure 4. Cross spectral densities $P_{x z}$ of all 17 flight paths averaged in wave number bins. 

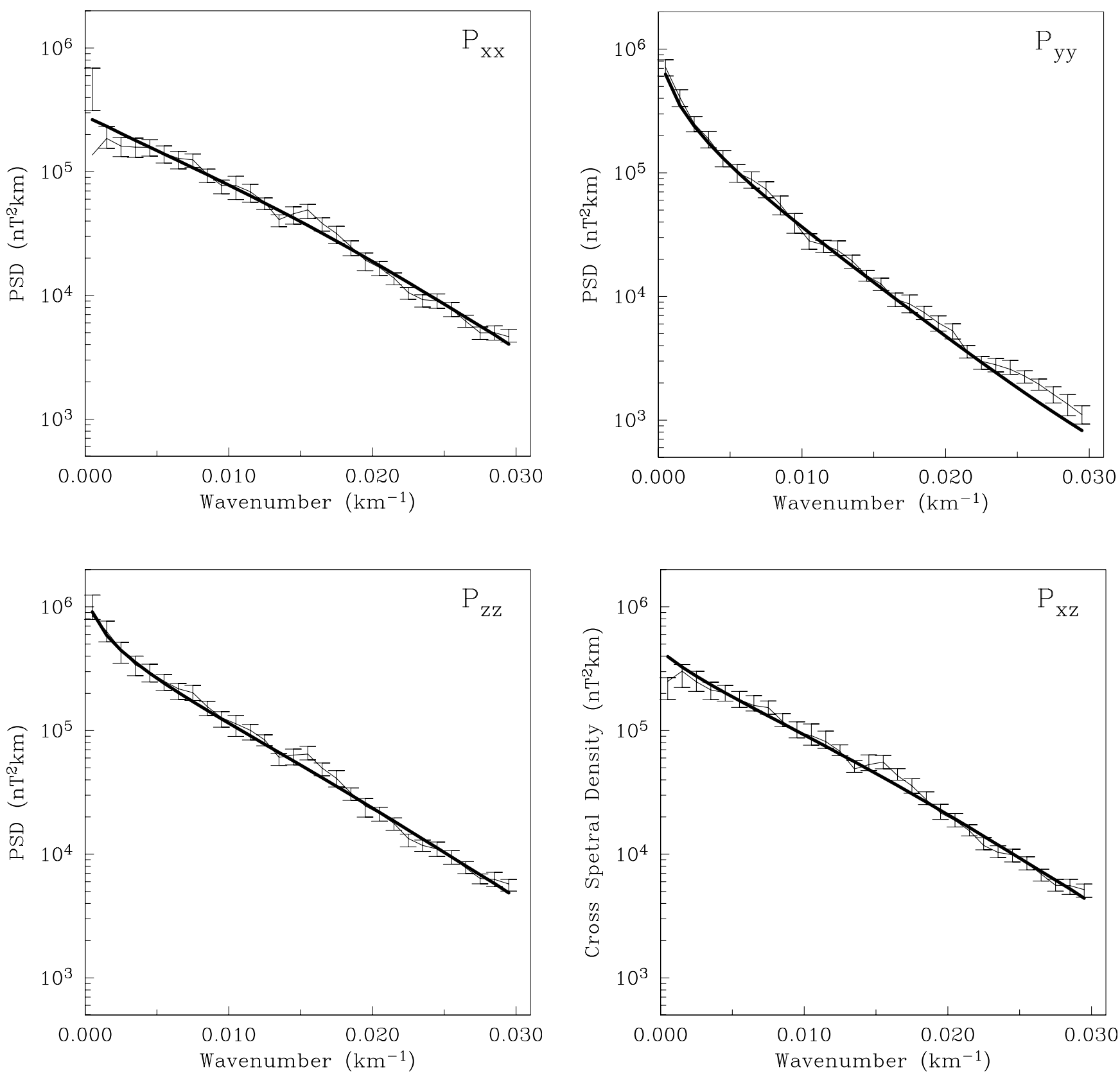

Figure 5. Power and cross spectral densities averaged over all 17 flight paths in wave number bins, shown with $\pm 1 \sigma$ uncertainty estimates. The thin line is the prediction of the optimally fitting model, and the thick line is that of the model smoothed within the uncertainties, see section 4 and Figure 6.

constraint on the correlation function of the magnetization. Considering the Earth, it seems reasonable to require the correlation of the crustal magnetization to decrease with distance and even to be zero for sites that are separated by great angles. On the basis of this approach we attempt to find a more reasonable behavior of our model for the very long wavelengths.

[14] The correlation function $C(\mu)$ can be represented as a Legendre expansion

$$
C(\mu)=\sum_{l=0}^{\infty} c_{l} P_{l}(\mu)
$$

where the $P_{l}(\mu)$ are the Legendre polynomials, $\mu$ being again the cosine of the colatitude $\theta$ along the great circle path.
Jackson [1994] showed that the coefficients $c_{l}$ are related to the power spectrum by

$$
R_{l}=\frac{\mu_{0}^{2}}{r_{0}^{2}} \frac{l(l+1)}{2 l-1} K(l, \epsilon) c_{l-1}, \quad l \geq 1,
$$

where

$$
K(l, \epsilon)=\left[1-(1-\epsilon)^{2 l+1}\right]
$$

Here, $\epsilon=d / r_{0}$, the scaled thickness of the magnetized crust. A good average value for the whole Earth is $d=35 \mathrm{~km}$. We are mainly dealing with oceanic crust in our data, however, 


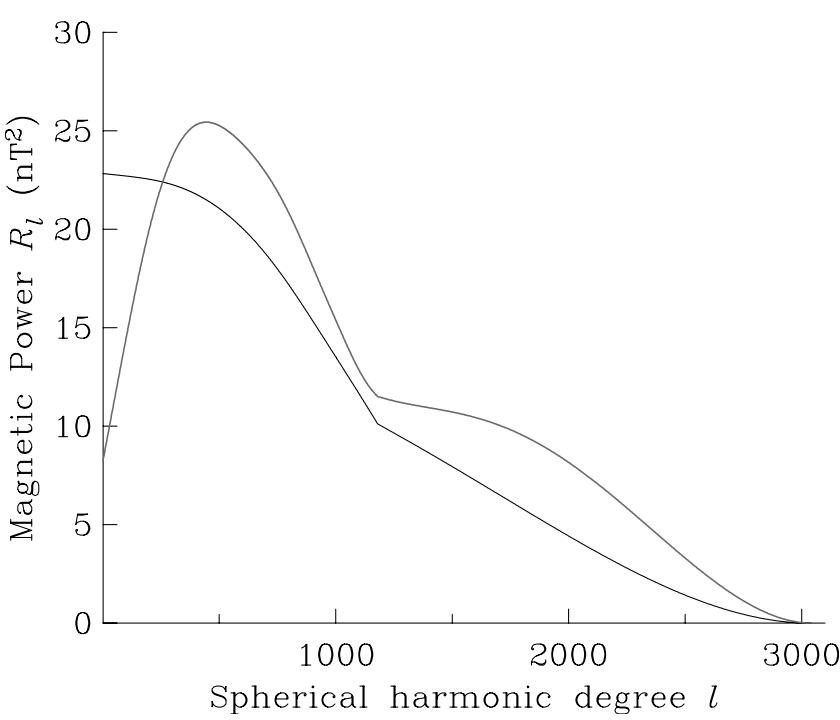

Figure 6. Smooth crustal geomagnetic power spectrum regularized by its second derivative (black). The model is well constrained by data in the range $l=20$ to 1200 , for smaller and higher spherical harmonic degrees the model is dictated by the smoothing constraint. Gray line is $O^{\prime}$ Brien et al.'s [1999] model, which is well-constrained by data in the range $l=60$ to 1200 .

so we chose a smaller value of $d=15 \mathrm{~km}$. Getting a direct relation between the coefficients $c_{l}$ and the power and cross spectral densities is straightforward: we simply have to plug equation (8) into equations (2) to (5). The coefficients $c_{l}$ have to be positive because of equation (8), so we can set up a NNLS problem like equation (6) again. The vector $\mathbf{x}$ now consists of the $c_{l}$ and $A$ is the matrix of the modified factors. We want to use the constraint that the correlation function is decreasing monotonically with distance, $d C(\mu) / d \mu \geq 0$. According to equation (7) this can be written as $D \mathbf{x} \geq 0, D$ being a matrix of the derivatives of the Legendre polynomials with respect to $\mu$. The constraint now can be included in the NNLS problem using positive slack variables [Stark and Parker, 1987] and suitable weighting of the data fitting part. We choose the number of $\mu_{i}$ over the interval $[-1,1]$ to be 500 , with asymptotically denser spacing toward the borders of the interval where the Legendre polynomials are more oscillatory than in the middle. A comparison with larger numbers of $\mu_{i}$ shows that we have reached convergence with our choice.

[15] We again have a highly underdetermined system of linear equations. We had hoped for the correlation constraint to force a smooth model when fitting the data to the uncertainties, $\chi^{2}=N$. However, it turns out that this is not the case. Although the obtained correlation function looks sensible, being high for small separation, decreasing first very rapidly and then gradually going to zero, the respective geomagnetic spectrum model shows high spikes for the degrees $l$ corresponding to the data frequency bins and very low values in between. A look at the fit of the model to the data shows that the data are still overfitted for the high frequencies, the misfit in the order of the uncertainties is produced by large misfits for the long wave- lengths. The monotonicity of the correlation functions seems to be a very weak constraint.

[16] Apparently, we still need to apply some kind of additional smoothing to obtain a smooth geomagnetic spectrum. However, in that case we would not have any physical or statistical indication on how to weight the different constraints in order to obtain the desired fit. Therefore we tried an alternative approach: we use our smooth model obtained in the last section as input data and invert for the $c_{l}$. Obviously, from equation (8) we can directly calculate the $c_{l}$ and thus $C(\mu)$ from the geomagnetic spectrum. Doing this shows that the resulting correlation function, though never reaching zero, already decreases monotonically with increasing separation: an inversion with the monotonicity as additional constraint seems to be futile. However, as we stated in section 4, our model is well constrained by data only for a limited range of spherical harmonic degrees. Consequently, in this new inversion we should only use that part as input data. We do not care about the short-wavelength part here, as changes in one range of input data mainly affect that same range of the new output model. The lowest degree corresponding to the first wave number bin of the data was $l=20$. We possibly should not trust the data in that bin too much, however, as they are averaged over considerably fewer spectral estimate data than those in the other bins. Remember that the power sum rule was fulfilled for many of the paths only for frequencies falling in the second and higher bins. So plausible cutoff degrees for the reliable data lie between $l=20$ and $l=40$, corresponding to the first and second wave number bin of the spectral estimates, respectively. Somewhat arbitrarily, we choose $l=30$ for our final model, arguing that while the first wave number bin might not be fully reliable it should not be completely wrong and together with the expected smoothness the range of welldetermined spherical harmonic degrees should extend further down than to $l=40$. Figure 7 shows the correlation function $C(\theta)$ determined by that inversion and the subsequent new geomagnetic spectrum model. While we fit the input $R_{l}$ data perfectly $\left(\chi^{2}=0\right)$, the new correlation function now is high only for short separations and the new spectrum model shows a sensible increase in the power of the very long wavelengths. In section 6 we will discuss these results and compare both the model and its associated correlation function to the previous ones described in section 1 .

\section{Results and Discussion}

[17] We have developed an enhanced geomagnetic spectrum of crustal magnetization from a set of aeromagnetic flight path data already studied by O'Brien et al. [1999] by improving the treatment of external variations in the data. In contrast to O'Brien et al. we are able to fit the spectral estimates from the aeromagnetic anomaly data very well without having to discard any long-wavelength parts of the power and cross spectral densities. O'Brien et al. [1999] ascribed their failure to fit the data to a contamination of the data by external fields, which could not be eliminated in the data processing. We come to a different conclusion. There certainly are residuals of external variations in our data, too. With the CMP31 model we have taken out the external variations for magnetically quiet conditions, but we have no 
a

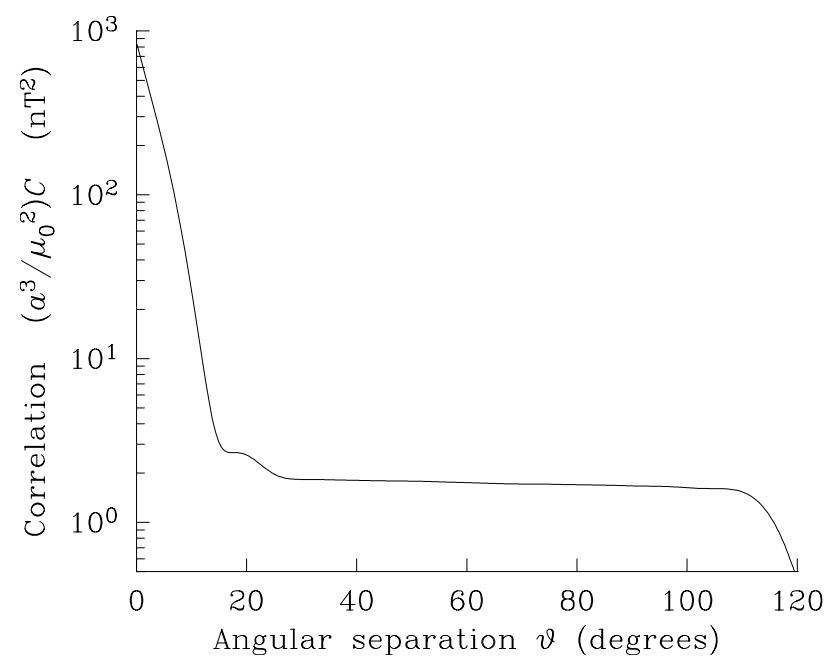

b

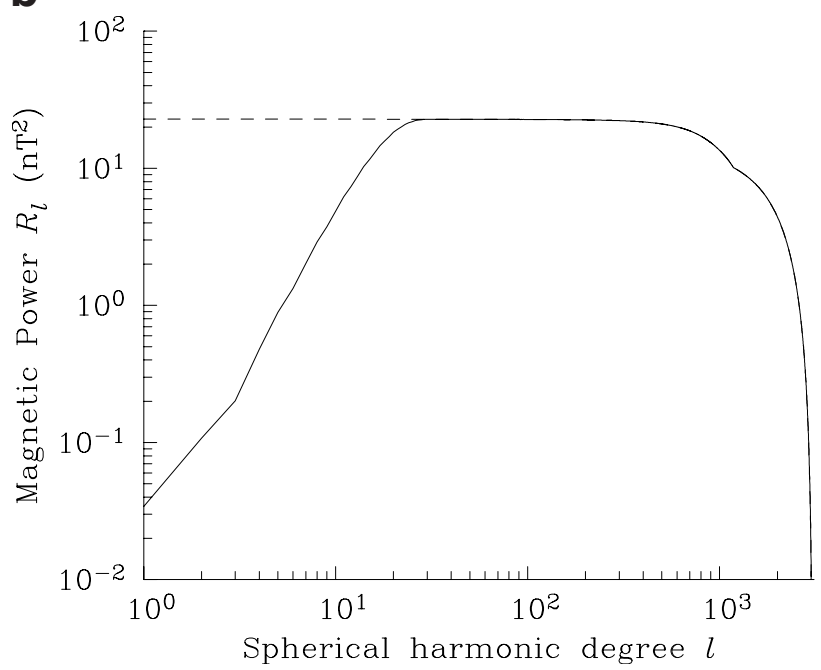

Figure 7. (a) Correlation function determined from the smoothed geomagnetic spectrum with $l \geq 30$ and subject to a monotonicity constraint. (b) New geomagnetic spectrum derived from that correlation function (solid line) compared to former spectrum (dashed line, Figure 6), plotted in logarithmic scale now.

control whatsoever over the infrequent variations during more disturbed conditions, which also occurred during the times of the measurements. These residuals, however, do not seem to be much of a problem, they obviously are averaged out quite well. Otherwise the power sum rule and the phase criterion would not have been satisfied so well. The problem with O'Brien et al.'s data rather is, that by subtracting cubic polynomials as representation of the external variations, they in fact have taken out too much. The slightly lower power for the long wavelengths suggests that they must have removed some crustal signal information. Moreover, the removal of distinct polynomials from the individual components is inconsistent and results in unnecessary violations of the stochastic model. Figure 8 compares the reliable part of our spectrum with earlier spectra based on spherical harmonic analyses of satellite data or mathematical models. For spherical harmonic degrees $>200$ our model does not differ much from that of O'Brien et al. [1999], while for the lower degrees it displays higher power agreeing better with other models than O'Brien et al.'s. Overall, our model agrees very well with the simple theoretical model of Langel et al. [1989], although for $l<30$ it agrees better with the satellite-derived models. Like our model these do not imply the unlikely high degree of correlation for very long wavelengths, seen in Langel et al.'s (see Figure 9). In our model the range of the increase of the spectrum at the very long wavelengths is determined by the choice of cut-off spherical harmonic degree for reliable data. The model reaches the level of its extended maximum generally at the first reliable input point of $l$. This is not surprising, as the correlation function offers only such a weak constraint and in fact is zero for large separations. However, there is no better way to bound this part of the spectrum than to make a reasonable guess about the reliability of the smooth model. The spectral estimates were obtained from flight paths of limited length, so they cannot contain this very long wavelength information on their own. Moreover, we must not forget another uncertainty about the long-wavelength part: By subtracting the model main field data from our raw data we might also have taken out very long wavelength crustal anomaly information, and also might have left residuals of the core field in the data. With our choice of minimum reliable degree $l=30$ our model agrees well with recent satellite-based estimates that should offer more reliable information about those long wavelengths.

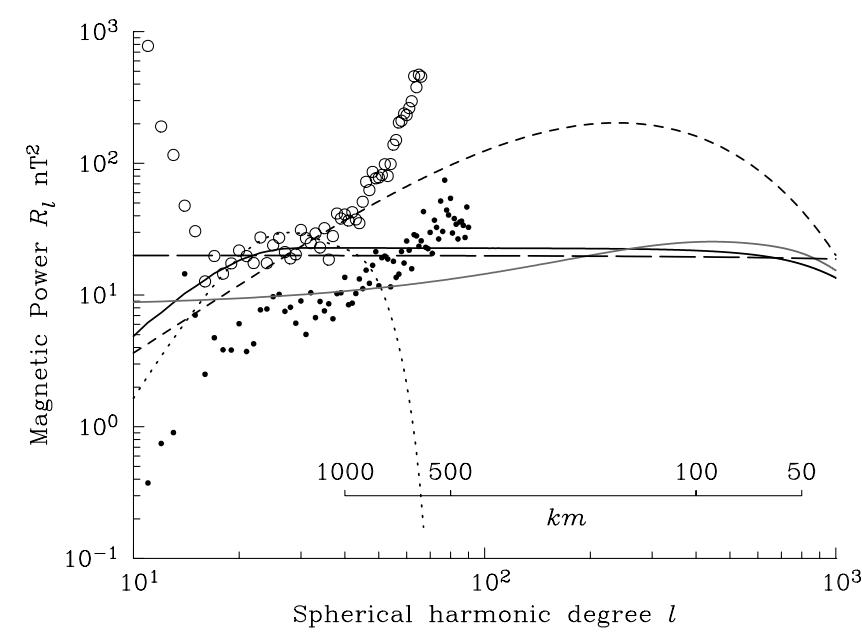

Figure 8. Different models of the geomagnetic power spectrum. Theoretical models by Jackson [1994] (short dashed line) and Langel et al. [1989] (long dashed line). Models based on satellite data by Cain et al. [1989] (circles), Ravat et al. [1995] (dots), and Lowe et al. [2001] (dotted line). Models from large-scale aeromagnetic data by O'Brien et al. [1999] (gray line) and this paper (solid black line). 


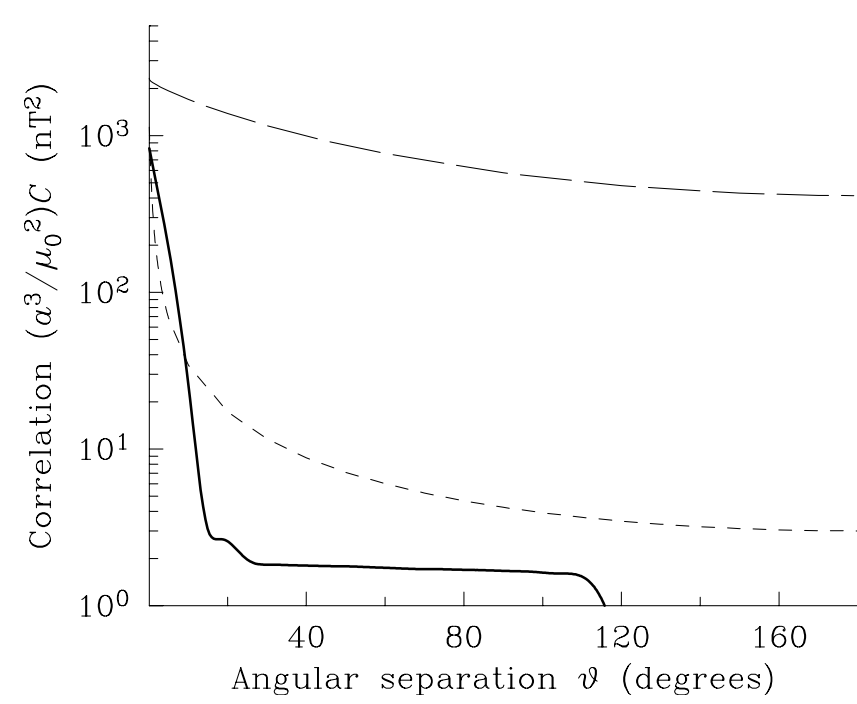

Figure 9. Comparison of our correlation function (solid line) with those corresponding to the models of Jackson [1994] (short dashed line) and Langel et al. [1989] (long dashed line).

[18] For degrees $l>50$ to 60 our model seems to lack power in comparison to the spherical harmonic analysis models and the theoretical model of Jackson [1994]. One reason might be that we only used oceanic crust data. Lowe et al. [2001] concluded that the oceanic crustal spectrum contains less power than the continental one due to the smaller volume of oceanic crust. However, Lowe et al.'s model itself does not contain more power. On the contrary, this model, stated to be reliable up to degree $l=45$ agrees remarkably well with ours. Note also that the spherical harmonic models, although plotted up to higher degrees, are stated to be reliable only up to $l=50$ [Cain et al., 1989] and $l=65$ [Ravat et al., 1995]. Considering this, our spectrum is in good general agreement with all of the satellite based models. Thus it can be seen as a real extension of the satellite spectra to higher spherical harmonic degrees, without leaving a gap of unreliable estimates in the range of wavelengths.

[19] The correlation function going along with our model (Figure 9) also looks highly satisfying. It is high only for short separation distances and becomes very low at about $\theta=20^{\circ}$. That means correlation of the magnetization is given only for separations smaller than approximately 2200 $\mathrm{km}$. Correlation functions associated with the theoretical models of Jackson [1994] and Langel et al. [1989] have been calculated by Rygaard-Hjalsted et al. [1997]. Comparing our result to those, it agrees well with that of Jackson [1994]. Our function just displays a sharper distinction between separation angles of high and insignificant correlation. The value of $20^{\circ}$ looks sensible for any correlation, rather too high than too low, considering that the crust generally is broken into small geological units. Thinking about the fine-scale structure of the magnetized bands of the ocean floor, the value might seem too high for our purely oceanic data. We must remember, however, that our profiles went in all directions. While some go almost perpendicular to the bands, others lie almost parallel and can display correlated magnetization over long distances. A correlation over separations of $180^{\circ}$, that is half the Earth or more as implied by the Langel et al. [1989] model seems highly unlikely. The correlation limit for our model is almost independent of the minimum degree of reliable estimate we use as input for that inversion, therefore it can be regarded as reliable. We should not forget, however, that it might be influenced by the limited length of our flight tracks and residuals of the core field or missing crustal signal from the removal of the main field model.

[20] Acknowledgments. This work was funded by NASA grant NAG5-7612 and partly by Alexander von Humboldt-Foundation (FeodorLynen fellowship to M.K.). We wish to thank Jerome Dyment for a detailed and constructive review. We also thank Joseph Cain and Maurice Tivey for their reviews of the original manuscript.

\section{References}

Bloxham, J., D. Gubbins, and A. Jackson, Geomagnetic secular variation, Philos, Trans. R. Soc. London, Ser. A., 329, 415-502, 1989.

Cain, J. C., Z. Wang, C. Kluth, and D. R. Schmitz, Derivation of a geomagnetic model to $n=63$, Geophys. J., 97, 431-441, 1989.

Hittelmann, A. M., R. W. Buhmann, and S. D. Racey, Project Magnet aeromagnetic surveys 1954-1994, U.S. Dep. of Commer., Natl. Oceanic and Atmos. Admin., Natl. Geophys. Data Cent., Boulder, Colo., 1996.

Jackson, A., Accounting for crustal magnetization in models of the core magnetic field, Geophys. J. Int., 103, 657-674, 1990.

Jackson, A., Statistical treatment of crustal magnetization, Geophys. J. Int., 119, 991-998, 1994.

Jackson, A., Bounding the long wavelength crustal magnetic field, Phys. Earth Planet. Inter., 98, 283-302, 1996.

Langel, R. A., R. H. Estes, and T. J. Sabaka, Uncertainty estimates in geomagnetic field modeling, J. Geophys. Res., 94, 12,281-12,299, 1989.

Lawson, C. L., and R. J. Hanson, Solving Least Squares Problems, Prentice-Hall, Old Tappan, N. J., 1974.

Lowe, D. A. J., R. L. Parker, M. E. Purucker, and C. G. Constable, Estimating the crustal power spectrum from vector Magsat data, J. Geophys. Res., 106, 8589-8598, 2001.

McLeod, M., and P. J. Coleman, Spatial power spectra of the crustal geomagnetic field and core geomagnetic field, Phys. Earth Planet. Inter., 23, 5-19, 1980 .

Meyer, J., J. Hufen, M. Siebert, and A. Hahn, Investigations of the internal geomagnetic field by means of a global model of the Earth's crust, J. Geophys., 52, 71-84, 1983.

O’Brien, M. S., R. L. Parker, and C. G. Constable, Magnetic power spectrum of the ocean crust on large scales, J. Geophys. Res., 104, 29,18929,201, 1999.

Parker, R. L., Geophysical Inverse Theory, Princeton Univ. Press, Princeton, N. J., 1994.

Parker, R. L., and M. S. O'Brien, Spectral analysis of vector magnetic profiles, J. Geophys. Res., 102, 24,815-24,824, 1997.

Ravat, D., R. A. Langel, M. Purucker, J. Arkani-Hamed, and D. E. Alsdorf, Global vector and scalar Magsat magnetic anomaly data, J. Geophys. Res., 100, 20,111-20,136, 1995.

Riedel, K. S., and A. Sidorenko, Minimum bias multiple taper spectral estimation, IEEE Trans. Signal Process., 43, 188-195, 1995.

Rygaard-Hjalsted, C., C. G. Constable, and R. L. Parker, The influence of correlated crustal signal in modelling the main geomagnetic field, Geophys. J. Int., 130, 717-726, 1997.

Sabaka, T. J., R. A. Langel, R. T. Baldwin, and J. A. Conrad, The geomagnetic field 1900-1995, including the large-scale field from magnetospheric sources, and the NASA candidate models for the 1995 revision of the IGRF, J. Geomagn. Geoelectr., 49, 157-206, 1997.

Sabaka, T. J., N. Olsen, and R. A. Langel, A comprehensive model of the near-Earth magnetic field, NASA Tech. Memo., TM-2000-209894, Goddard Space Flight Cent., Greenbelt, Md., 2000.

Stark, P. B., and R. L. Parker, Velocity bounds from statistical estimates of $\tau(p)$ and $X(p)$, J. Geophys. Res., 92, 2713-2719, 1987.

C. G. Constable, M. Korte, and R. L. Parker, Institute of Geophysics and Planetary Physics, Scripps Institution of Oceanography, University of California, San Diego, 9500 Gilman Drive, La Jolla, CA 92093-0225, USA. (mkorte@igpp.ucsd.edu) 